\section{Sara Bigardi}

Università di Verona

sarabigardi@gmail.com

\section{Sizigia: spazio relazionale \\ e simbolico \\ Syzygy: A Relational and Symbolic Space}

Recepción: I 4 de noviembre de 2016

Aceptación: I 8 de diciembre de 2016

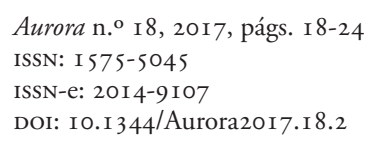

\section{Resumen}

\section{Abstract}

En este artículo se desarrolla el significado de la palabra syzyguía en María Zambrano. El término será analizado desde las cartas a Agustín Andreu y en referencia al manuscrito número 408 de i968. Lo que resulta interesante es ver cómo esta palabra reúne diferentes elementos y abre espacios de sentido que toman en consideración la hermandad, la amistad, el sueño compartido y la pequeña comunidad creada por afinidad metafísica.

\section{Palabras clave}

Syzyguía, comunidad, paredro, amistad, encuentro, metafísica, lugares, Ángel, sueño compartido
This article describes the meaning of the word syzyguia («syzygy») in Maria Zambrano. The term is analyzed from its appearance in the letters addressed to Augustin Andreu and with reference to manuscript number 408 of I 968 . It is interesting to see how the word opens new thought spaces that take into consideration sisterhood, friendship, the shared dream, and the small community created around the affinity for metaphysics.

\section{Keywords}

Syzygy, community, paredros, friendship, encounter, metaphysics, places, angel, shared dream

La amistad corresponde a la meditación, a la vida de la conciencia: es caminar despierto juntos o al mismo tiempo. $\mathrm{Al}$ mismo tiempo, no en el mismo tiempo.

María Zambrano

Il pane deve essere mangiato in compagnia [...] non può essere mio soltanto, o di chicchessia. $\mathrm{O}$ è il nostro, e quindi di tutti, o non è di nessuno e allora il mangiarlo appare un'usurpazione (fatta non solo all'altro, ma anche all'essere del pane).

María Zambrano

'Sizigia' è una parola che ha sempre destato il mio interesse. Si tratta di un termine composto, la cui etimologia deriva dal latino syzygia e dal greco syzygos: "voce formata dal prefisso syn (con, unitamente, ma anche ciò che succede allo stesso tempo) più zygon (giogo, qualco- 
In Zambrano la parola 'sizigia' trova una precisa e puntuale apparizione che diviene tuttavia significativa in un quadro più grande. Per la qualità e il significato variegato con cui compare, non è propriamente un tema guida del suo pensiero, soprattutto se messa a confronto con altri temi che ricorrono con ben altra consistenza, diventando elementi cardine del suo filosofare. 'Sizigia' viene accennata, senza diventare mai oggetto di una trattazione sistematica.

La parola compare in Zambrano come titolo di un manoscritto del 1968, ${ }^{2}$ in alcune lettere indirizzate ad Agustín Andreu nello scambio epistolare che, iniziato nel ' 50 , si intensificò negli anni ' $70,{ }^{3} \mathrm{e}$ in un manoscritto intitolato Historia y poesía. ${ }^{4}$

Partendo da questi precisi contesti, il lavoro si articola seguendo due prospettive. Da un lato si propone di aprire degli spazi di riflessione che nascono dalla relazione, anche simbolica, tra il termine 'sizigia', altre parole-chiave e alcuni temi guida del pensiero zambraniano; dall'altro, propone una lettura che cerca di interpellare il contemporaneo, facendo appunto leva sui nodi e le questioni che emergono da questo argomentare.

Mi muovoquindi seguendo, in primis, la corrispondenza tra Zambrano e Andreu, raccolta nel volume Cartas de La Pièce: è in questo percorso dialogico che la parola 'sizigia' acquista una certa consistenza tematica. Tale inizio è fertile anche dal punto di vista metodologico. Secondo le diverse accezioni proposte da Zambrano, il metodo, inteso come «via», cammino, ${ }^{5}$ ha a che vedere innanzitutto con il tempo. ${ }^{6}$ Anzi, potremmo dire, con una «molteplicità di tempi» da tenere insieme allo stesso tempo. In questo caso si tratta del tempo storico degli anni '70, di quello della scrittura, in questo caso la scrittura delle lettere all'amico teologo, e di un tempo-spazio preciso, quello de La Pièce, ${ }^{7}$ una località tra la Francia e la Svizzera dove Zambrano viveva con la sorella Araceli in una casa in mezzo al bosco.

Fare luce su questo, permetterà anche di prendere in considerazione, in un intreccio plurale, a più livelli, alcuni passaggi di un manoscritto che ha come titolo: Syzyguía. Perché il metodo è anche questo, «un luogo di arrivo [...], luogo di convivenza quindi».8

\section{La sizigia nelle lettere ad Andreu}

Zambrano ha scritto molte lettere nella sua vita: i suoi epistolari sono luoghi in cui, per usare una sua espressione, c'è «un'assistenza alla verità a partire dalla realtà che incalza». ${ }^{9}$ Assistere è vedere $\mathrm{e}$ accogliere la verità nello «spazio dell'anima intera, psiche, coscienza con tutti i suoi attributi di volontà e responsabilità», ${ }^{10}$ a partire dalla realtà con cui dobbiamo necessariamente "trattare». ${ }^{.1}$
I. Etimologia di 'sizigia': http://etimologias. dechile.net/?sizigia. Le sottolineature sono mie.

2. M-408: Syzyguía, 1968.

3. Zambrano, M., Cartas de La Pièce (Correspondencia con Augustin Andreu), Valencia, Pre-Textos, Universidad Politecnica, 2002, pp. 37-43 (carta $\left.\mathrm{n}^{\circ} 4\right), 45$ (carta $\mathrm{n}^{\circ}$ 5), I2I-I25 (carta n $\left.{ }^{\circ} 26\right)$, I67-I7I ( $\left.\operatorname{carta~n^{\circ }} 40\right)$ e pp. 350-352. Oltre a queste quattro lettere ce ne sono altre che fanno indirettamente riferimento al tema.

4. M-214: Historia y poésia (1974-1978).

5. "La riflessione sul metodo ha accompagnato tutta la ricerca filosofica di Zambrano. Ricondotta al suo significato letterale di "via oltre», cammino che conduce al di là, la parola 'metodo' rappresenta per la filosofa il percorso di una vita: dentro uno spazio unitario, che comprende sia lo spazio fisico dell'agire (esterno) sia lo spazio intimo della coscienza con le sue modificazioni. Nel metodo così inteso, agire, sentire e pensare cominciano a integrarsi, s'impara facendo», in Savelli, G., María Zambrano e il sogno del divino femminile, introduzione di Chiara Zamboni, Roma, Iacobelli Editore, 20I4, p. I3I.

6. Zambrano, M., Cartas de La Pièce (Correspondencia con Augustin Andreu), cit., p. 37.

7. Attraverso gli scritti di Zambrano ho immaginato questo luogo, la casa, chiamata da María in vari modi (convento abandonado, choza, nido, cenobio, granja, catacumba, gruta, cámara de tortura, jaula, madriguera), il bosco, i suoi chiari, la luce, la notte. Ma La Pièce rimane per me qualcosa di misterioso.

8. Zambrano, M., Note di un metodo, a cura di S. Tarantino, Napoli, Filema Edizioni, 2008, p.37.

9. Questa frase si trova ne Il sogno creatore, precisamente in un saggio intitolato «Il sonno dei discepoli nell'Orto degli Ulivi», ed è riferita al luogo dell'Uomo, quello che i discepoli, addormentandosi, hanno abbandonato: «E sprofondando nel sonno lasciarono incustodito il luogo dell'Uomo: tempo, libertà, assistenza alla verità a partire dalla realtà che incalza». Zambrano, M., Il sogno creatore, traduzione di V. Martinetto, Milano, Bruno Mondadori, 2002, p. 172.
Io. Ivi, cit., p. 179 .
II. Ivi, cit., p. 62 
I2. Buttarelli, A., «Femminismo radicale», in Diotima, Femminismo fuori sesto. Un movimento che non può fermarsi, Napoli, Liguori, 2017, pp. 48-49. La sottolineatura è mia.

I3. La questione teologico-metafisica è stata bene trattata da L. Vantini nel libro La luce della perla. La scrittura di Zambrano tra filosofia e teologia, Torino, Effatà Editrice, 2008, e in un articolo di J. Sánchez-Gey Venegas, «El pensamiento teológico de María Zambrano: Cartas de La Pièce. Correspondencia con Agustín Andreu», pubblicato nel numero i6 di Aurora. Papeles del «Seminario María Zambrano» del 2015, pp. IO4-II3.

I4. Andreu, A., «Preliminares a esta edición», in Zambrano, M., Cartas de La Pièce (Correspondencia con Agustín Andreu), cit., p. 2I.

I5. «Dire non presuppone un'azione, e meno ancora un qualcosa, ma un qualcuno; qualcuno che ascolti quando ancora non si sa cosa si dirà...trascendendo la finalità», Zambrano, M., Dell'Aurora, traduzione di E. Laurenzi, Genova, Marinetti I820, 2000, p. 88.

I6. Vantini, L., La luce della perla. La scrittura di Zambrano tra filosofia e teologia, cit., pp. 24-25.

17. Andreu, A., "Anotaciones epilogales a un método o camino", in Zambrano, M., Cartas de La Pièce (Correspondencia con Agustín Andreu), cit., p. 350.
Nelle lettere Zambrano dà conto di ciò che per lei è irrinunciabile, di ciò che le sta più a cuore. Sono un momento soggettivo e di ricerca insieme: un partire da sé. Da un punto di vista intimistico lei è in gioco in questo: c'è un elemento di autenticità. "L'autenticità — scrive Annarosa Buttarelli — è sinonimo di radicalità [...]. Quando si lavora per capire se c'è radicalità in ciò che facciamo, pensiamo o inventiamo, bisogna che guardiamo come mettiamo in forma le nostre relazioni e le conseguenze che discendono da questo processo». ${ }^{12}$

Le lettere di Zambrano, e nella fattispecie quelle ad Andreu, sono un concentrato di autenticità e radicalità: ovvero vanno osservate attraverso la misura della relazione che mettono in forma e presenza.

È vero che il tema centrale di questa corrispondenza è la riflessione teologico-metafisica, ${ }^{13}$ della circolazione del Logos e dello Spirito e la conoscenza della Trinità, ma è altrettanto vero, come dice Andreu, che «el epistolario trasunta hasta el clima doméstico de Roma y de La Pièce. La universidad, san Augustín, la naturaleza, la gnosis, el matrimonio, la syzyguía o comunidad breve, la amistad, el exilio, los Maestros, la Razón Vital..., todo el mundo de María aflora aquí».14 E dire che "aflora todo el mundo de María» significa affermare che appare tutto il suo esserci per che si incardina nella relazione di un dire: ${ }^{55}$ nello svelamento a qualcuno di ciò che accade e si vive. In questo dire che non presuppone propriamente un'azione, ma un chi, un qualcuno, c'è l'altro fondamentale elemento di queste lettere: la sizigia, che in questo percorso dialogico è al contempo metaparola, metadiscorso e tema.

Innanzitutto la corrispondenza con Agustín Andreu è essa stessa uno "spazio relazionale», concepito da Zambrano come sizigia, «in cui si condivide o si trasmette un'esperienza di ricerca (è la dimensione del syn) che si rivela ispirata e curata da un'istanza obbligante, trascendentale e immanente allo stesso tempo (è la dimensione del zygon, del giogo che riguarda entrambi)». Le lettere sono quindi già una sizigia e prendono così la forma di un discorso sul discorso.

Lo spazio relazionale assume allora diverse connotazioni, tutte affini tra loro, a secondo di come viene declinata la parola 'sizigia'. Sizigia è un sogno condiviso che protegge da ogni solipsismo e autoreferenzialità, richiedendo "non solo un metodo comunionale», ma anche l'uscita da sé. ${ }^{16}$ Sizigia è «la comunidad pequeña (no comunista!), verdadera, la de la afinidad producida por experiencia metafísica», ${ }^{17}$ è una relazione di amicizia presente ma soprattutto accudente, un rapporto discepolare, un paredro, un legame fraterno e/o filiale, una identificazione angelica, un legame tra sorelle, la sorellanza.

Di fronte alla poliedricità semantica, e non solo, della parola 'sizigia', occorre pensare a un taglio sui tanti significati e aspetti da mostrare. Stare in questa prospettiva significa rispondere a domande essenziali, 
nella consapevolezza che è impossibile verificare ogni risposta su questo tema. Vorrei allora soffermarmi sul sogno condiviso, sulla piccola comunità prodotta e creata per esperienza metafisica, sull'amicizia, il legame tra lei e Araceli, la sorellanza. E parto proprio da quest'ultima.

\section{Un caso di «identificazione angelica»: il legame con Araceli e la sorellanza}

\section{Scrive Agustín Andreu:}

La syzyguía de la Zambrano eran ella y Mariano, su primo hermano, de quien cosas tan bellas ha dicho. Pero ella buscó incansablemente la demostración experimental de que la syzyguía, la pequeña comunidad (no comunista!, dice) verdadera, la de la afinidad producida por experiencia metafísica, es posible. No me refiero aquí a Araceli, porque no era sólo un caso de syzyguía, sino de identificación angélica. ${ }^{18}$

A La Pièce Zambrano viveva con suo cugino Mariano e la sorella Araceli. I tre formavano una piccola comunità: «un numero tre» che sigillò il reale di quel momento fino alla morte della sorella, nel ' 72 .

La sizigia con la sorella, ${ }^{19}$ questa sorta di paredro indissolubile, simbiotico, una identificazione angelica, che unisce l'inizio e la fine, la vita e la morte, ${ }^{20}$ dà la misura di quanto è essenziale e determinante per il pensiero di Zambrano lo «spazio condiviso».

Sizigia e identificazione angelica allo stesso tempo. Non è un caso che il manoscritto 408 del 1968, intitolato appunto Syzyguía, contenga molte pagine dedicate al tema dell'Angelo, figura di mediazione per eccellenza, ${ }^{21}$ che Zambrano scrive sempre con la a maiuscola. Questo manoscritto, difficile da decrittare, soprattutto per la scrittura stilizzata, richiederebbe un preciso lavoro filologico. Da una sommaria decifrazione, ciò che emerge in riferimento all'Angelo sono tre aspetti che hanno a che vedere con la fiducia, l'incontro e il luogo. L'intelligenza angelica è ciò che, presupponendo «confianza en tu propia alma», muove, dal luogo in cui ci si trova (Zambrano dice dal «lugar donde el Ángel nos deja») all'incontro: un incontro che non si vuole, ma si cerca.

In un' intervista rilasciata per la televisione spagnola, ${ }^{22}$ Zambrano dichiarò che da Araceli aveva imparato la cosa più importante della vita, più importante anche della libertà: la hermandad.

Ahh! È mia sorella. La mia unica sorella. Scrivendo Antigone, io andai al termine greco 'autoadelfos', perché adelfa [oleandro] significa sorella. E curiosamente nei giardini di Segovia se ne piantavano sempre due, una rossa e una bianca. Mia sorella, mia sorella unica. Come l'ho aspettata! Perché nacque quando io avevo sette anni. Che gioia avere

\section{Ibidem}

19. Zambrano, dopo il 1939, anno che segna il suo esilio, raggiunge Araceli a Parigi solo nel I948, un giorno dopo la morte della loro madre. Rimangono in Francia un anno e poi partono per Cuba, L'Avana, dove rimangono fino al '53. Ritornano in Europa, a Roma, e ci rimangono fino al ' 64 e da lì a La Pièce, dove Zambrano vivrà fino al suo ritorno in Spagna.

20. "Così la vidi un'ora dopo la sua nascita. C'era un'adeguazione perfetta, era la stessa creatura, solo creatura di nuovo, innocente, casta, maestosa ora, bellissima, come se la storia — di cui morì, poiché la sua sofferenza mortale fu la storia - non fosse esistita, intatta, fragrante, quasi luminosa di luce propria. La forma, la sua, pura, perfetta, e la fragranza erano di totale presenza»,

Zambrano, M., La Cuba secreta y otros ensayos, Madrid, Endymion, 1996,

pp. 224-225.

«Araceli conservó intacta su fragancia hasta el último instante en medio del 'double delire de double depression nerveuse'», Zambrano, M., Cartas de La Pièce, cit., p. I07.

2I. L'Angelo non si presenta mai "por sí o en sí, sino en figuras muy variables. Incluso en tu madre, en tu maestro, en tu bienhechor, en tu amigo [...] dejando caer un par de palabras a quién sabe quién». Andreu, A., "Anotaciones epilogales a un método o camino", in Cartas de La Pièce, cit. pp. 349-350.

22. Mandata in onda nel programma «Muy personal» della Televisión Española. Trascrizione di M. M. Rivera Garretas, Duoda. Revista de Estudios Feministas, ${ }^{\circ} 25$, 2003. 
23. Ibidem, traduzione di C. Jourdan in Diotima, Archivio Per amore del mondo.

24. Per Andreu questo legame non va analizzato o interpretato secondo un registro psicologico, pena il travisamento del pensiero zambraniano, Cfr., Andreu, A., María Zambrano. El Dios de su alma, Albolote (Granada), Comares, 2007, cit., p. I46.

25. Zambrano, M., Cartas de La Pièce (Correspondencia con Agustín Andreu), Carta n. ${ }^{\circ}$, I9 de mayo de 1974. Domingo, cit., p. 4I.

26. Sánchez-Gey Venegas, J., «El pensamiento teológico de María Zambrano: Cartas de La Pièce. Correspondencia con Agustín

Andreu", cit., pp. I09-IIo. una sorella; con lei scoprii ciò che è più importante nella mia vita, la sorellanza, la sorellanza, più della libertà, la sorellanza. ${ }^{23}$

Questa relazione, di per sé orientante, orienta anche il modo in cui Zambrano concepisce la sizigia, in quanto è a partire da questo legame di sangue, e non solo, con Araceli, che si delineano gli aspetti che connotano la piccola comunità.

Prima di addentrami in questo, vorrei soffermarmi sulla sorellanza, che, come abbiamo visto, è per Zambrano la cosa più importante della vita, più anche della libertà. Questa è un'affermazione forte in vari sensi. Innanzitutto, questo rilevante riconoscimento della sorellanza non svalorizza la libertà, di cui in un tempo come quello vissuto dalla filosofa si sentiva costantemente la mancanza, anzi. Queste parole di Zambrano presuppongono che senza forme di co-esistenza, come la preziosa sizigia della sorellanza, la libertà perde importanza e autorità. Poi l'affermazione mette in luce altri due aspetti: esalta la potenza della sorellanza come relazionalità tra donne, che si iscrive in una precisa genealogia di sangue, quella naturale, che la vita ci ha dato in dono, e invita a non sostare nell'assoluta prevalenza del legame di sangue, ma a completarla.

La sorellanza per Zambrano supera infatti la pura affettività, sempre suscettibile di scadere in una sorta di infecondo sentimentalismo o in atteggiamento consolatorio e protettivo, ${ }^{24}$ per favorire un legame che, trascendendo entrambe, sfocia in una "comunidad de hermanos», così come Zambrano scrive in una lettera:

[En la syzyguía] en la que <tanto se refiere Massignon> vivieron San Juan y la Virgen y quizás Magdalena en Éfeso; la que tanto reprochan a San Pablo haber con Santa Tecla. Massignon dice que en ella está cifrada la prosecución de una vida espiritual en Occidente, lo que a Araceli y a mí nos llenó de júbilo. Pues que siendo enteramente femeninas, desde jovencitas tendíamos a una unión así, el matrimonio doméstico nos echaba para atrás. Y así seguimos cuando ya solas y todavía jóvenes y ella en estado de merecer, vivimos como... se sabe..., siéndonos el hombre, su trato, su amistad, su parentesco, indispensable, y fuente de las mayores riquezas del alma, espíritu y hasta de cuerpo, en la alegría. - Quiero decir en la vitalidad que nos daba el trato con los amigos. ${ }^{25}$

La sorellanza è il presupposto per la nascita di spazi condivisi, di piccole comunità, caratterizzate dalla vitalità di un incontro con gli amici, diventando quindi possibilità per la creazione di sizigie formate da "hombres verdaderos». "[...] Y es en esta vivencia de lo verdaderamente humano - scrive Sánchez-Gey Venegas — donde está la huella de lo divino, la que también le salva de los partidismos de los que siempre huyó [...] aunque sin alejarse nunca de lo real en su intimidad». ${ }^{26}$ Unioni quindi che rompono il solipsismo, l'autoreferenzialità e i legami di coppia esclusivi, per tracciare una «vía unitiva» che, attraverso la compenetrazione di intelligenze vere e 
Il legame tra Zambrano e Araceli si inserisce quindi in una ricerca simbolica, dove la forza originale, cioè originaria, di questo legame viene ritrovata nella figura di Antigone, una figura femminile che precede entrambe, rivelandole Araceli, la sua storia, il suo dolore. ${ }^{27}$ Si è scritto molto di Antigone alias Araceli, e quindi non entro nel merito di questo argomento. Lo porto solo come esempio di un'apertura simbolica e politica del loro legame ad altri legami.

Attraverso questo gesto, Zambrano si sceglie in qualche modo un'altra sorella e lo fa nella misura di un ascolto e di una accoglienza, reiventando la narrazione di sé e di Araceli in relazione ad altri. ${ }^{28}$ Per questo si può parlare della loro sorellanza in termini di una pratica politica e spirituale che, partendo da un percorso di libertà e radicalità femminile, ha dato vita alla condivisione di esperienze di vita e di pensiero con altri/e, formando comunità trasversali di amici $e$ amiche, legati tra loro da affinità metafisiche, e su cui potere contare in modo gioioso, allegro, accudente, passionale e anche conflittuale.

Viene così a crearsi, usando le parole di Federica Giardini, «una relazione di amicizia elettiva e basata sul principio delle singolarità soggettive». ${ }^{29}$

\section{Allo stesso tempo: l'amicizia, le affinità metafisiche e i sogni condivisi}

Mis amigos esenciales me están presentes siempre. Mas el que acudan me es también indispensable y luego, es gozo, alegría. ${ }^{30}$

L'amicizia per Zambrano corrisponde alla vita della coscienza, alla meditazione, cioè al «riconquistare il sentire originario delle cose, del paesaggio, della gente, degli uomini e dei popoli, il sentire della realtà immediata che ci apre la realtà del mondo».31 Amicizia è camminare insieme, non nello stesso tempo, ma allo stesso tempo, perché altrimenti sarebbe come vivere un tempo unico e identico a se stesso. Nello stesso tempo sono i tempi molteplici che accadono nell'incidenza della vita e che permettono il movimento dello stare nascendo, il movimento pendolare, come lo chiama Zambrano, «el más misterioso de todo lo que he percibido del fondo de mi ser». ${ }^{32}$

Molte sono state per lei le amicizie presenti e altrettante quelle accudenti, anche nella distanza. Sono state sizigie attente e incondizionate. Di quella «attenzione ardente, quella specie di respiro dell'essere, analogo al fiato degli animali che avevano assistito alla nascita terrestre».33 Queste sizigie, formate da «hombres enteros y verdaderos», cioè da esseri umani integri, ovvero autentici, cioè

27. «L'aveva chiamata Antigone, durante tutto quel tempo in cui il destino le aveva separate, tenendo lontana lei dal luogo della tragedia, mentre sua sorella Antigone, la affrontava. Cominciò a chiamarla così nella sua angoscia, Antigone, perché innocente sopportava la storia; perché nata per l'amore, la stava divorando la pietà». Zambrano, M., Delirio e destino, a cura di Rosella Prezzo, traduzione di Samantha Marcelli, Raffaelo

Cortina, Milano, 200o, p. 257.

28. Per un approfondimento della sorellanza si veda DWF (I07) Una Nessuna Centomila. Ancora sorelle?, 2015, 3.

29. Giardini, Federica, «Genealogie della socialità femminile», in Babelonline 2, p. 178.

30. Zambrano, M., Carta n. ${ }^{\circ}$ I, in Cartas de La Pièce, cit., p. 27.

3I. Zambrano, M., Delirio e destino, p. 86.

32. M-30: El movimiento pendular, 3 de septiembre 1955 .

33. Zambrano, M., «Il sonno dei discepoli nell'Orto degli Ulivi", in Il sogno creatore, cit., p. 173 . 
34. Cfr., Sánchez-Gey Venegas, «El pensamiento teológico de María Zambrano: Cartas de La Pièce», cit., p. Iıо.

35. Andreu, A., «Anotaciones epilogales a un método o camino", in Zambrano, M., Cartas de La Pièce (Correspondencia con Agustín Andreu), cit., p. 372.

36. Esposito, R., «Il dono della vita tra communitas e immunitas", reperibile online al link: siba-ese.unisalento.it/index.php/idee/ article/download/3356/276I

37. Andreu, A., «Anotaciones epilogales a un método o camino", in Zambrano, M., Cartas de La Pièce (Correspondencia con Agustín Andreu), cit., p. 343.

38. Zamboni, Chiara, «Introduzione» in Savelli, G., María Zambrano e il sogno del divino femminile, cit., p. 8. capaci di emanare la migliore energia di se stessi a partire dal «fondo insobornable» della loro radicalità umana, ${ }^{34}$ si sono create per affinità metafisica, avendo in comune un sogno condiviso.

Si delineano come piccole comunità non comuniste — in quanto il comunismo era per lei un movimento conservatore - né collettiviste. Sono una terza via che si propone di superare ogni posizione individualista e collettivista: «un mutuo jardín que inventan corazones al par», dove "se sale uno de sí hasta la frontera más próxima al otro [...] atravesando fronteras de humanidad».35 Come afferma Roberto Esposito, coloro che vivono la comunità «piuttosto che da una appartenenza, sono vincolati da un dovere di dono reciproco che li spinge a sporgersi fuori di sé, letteralmente a esporsi».$^{36}$

Le sizigie sono allora un sogno creatore condiviso, in cui si intersecano pratiche di vita, aspirazioni e progettualità. Un sogno che unisce e allo stesso tempo protegge dal solipsismo e dall'autoreferenzialità. Sono esperienze metafisiche che non ci tolgono dal tempo, ma ci fanno rimanere in esso, trasformandolo.

"La metafísica es experiencia del ser en los caminos del ser», ${ }^{37}$ ed è proprio in questa concezione della metafisica, che Zambrano impara da Antonio Machado, dove emerge anche l'aspetto qualitativo della metafisica stessa. L'essere umano, creatura mai nata del tutto, è colui che patisce un movimento costante verso l'essere, un movimento di trascendenza, che lo porta a sperimentare la vita. «Mentre un' esperienza è in corso - scrive Chiara Zamboni - i concetti metafisici si modificano, se esprimiamo quello che stiamo vivendo. Non sono dati in anticipo in modo statico, ma sono in circolo con il percorso di vita. Sono cardini orientanti l'esperienza». ${ }^{38}$ Lorientamento dato dalla metafisica sperimentale qualitativa è l'orizzonte in cui si creano le comunità basate sulle affinità conseguenti: «las syzyguías» in cui, come aveva intuito Massignon, è cifrata - e auspicabile aggiungo io - la prosecuzione di una vita spirituale, in cui convergono sorellanza, amicizia e partecipazione metafisica al sentire e al vivere comune. 


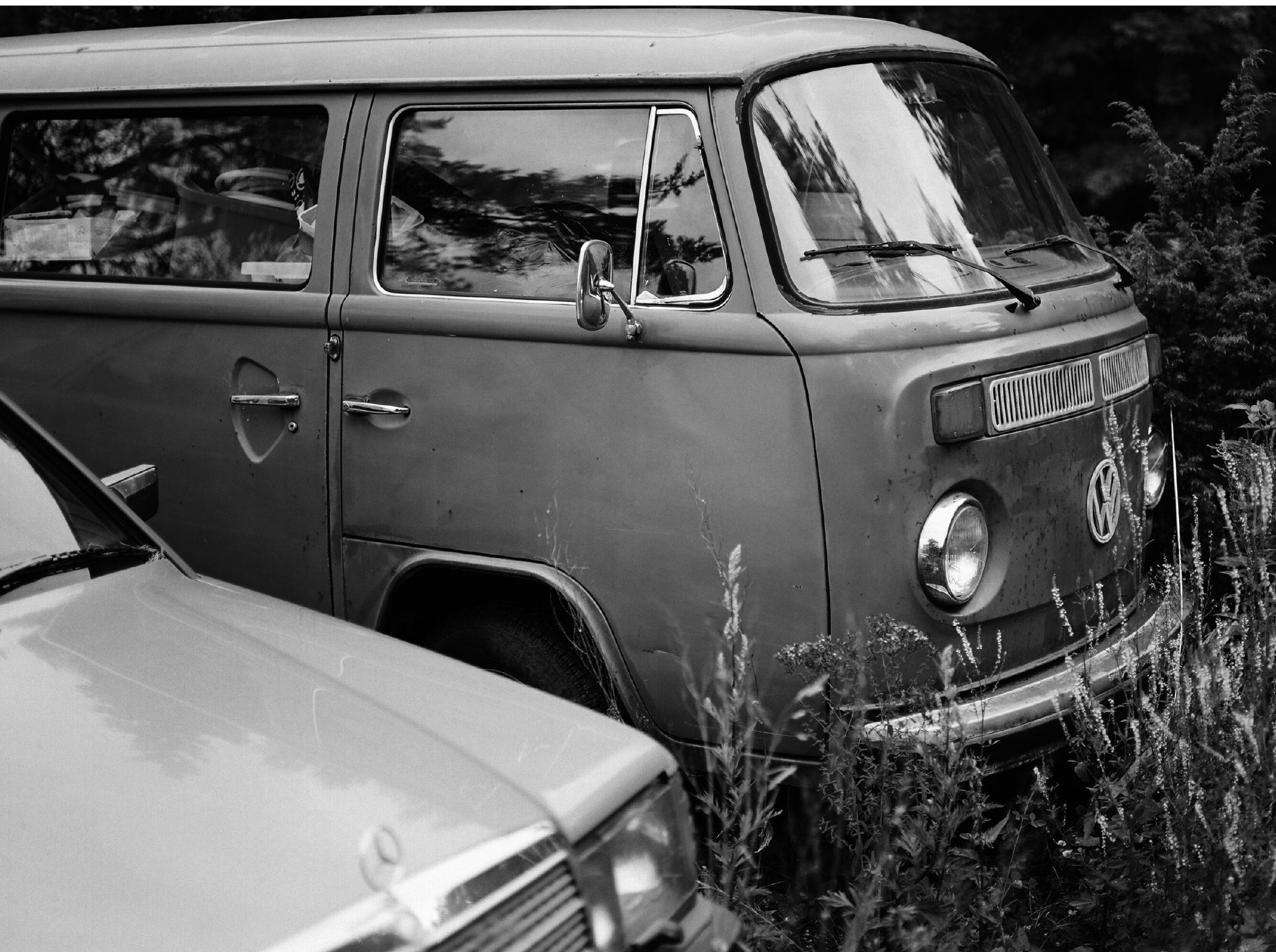

